\title{
The occurrence and risk factors of constipation in inpatient palliative care unit patients vs. nursing home residents
}

\author{
Tomasz Dzierżanowski ${ }^{1,2}$, Aleksandra Ciałkowska-Rysz ${ }^{2}$ \\ ${ }^{1}$ Laboratory of Palliative Medicine, Department of Social Medicine and Public Health, Medical University of Warsaw, Warsaw, \\ Poland \\ ${ }^{2}$ Laboratory of Palliative Medicine, Chair of Oncology, Medical University of Lodz, Lodz, Poland
}

Gastroenterology Rev 2018; 13 (4): 299-304

DOI: https://doi.org/10.5114/pg.2018.79809

Key words: constipation, palliative care, long-term care.

Address for correspondence: Tomasz Dzierżanowski MD, PhD, Laboratory of Palliative Medicine, Department of Social Medicine and Public Health, Medical University of Warsaw, 3 Oczki St, 02-007 Warsaw, Poland, e-mail: tomasz.dzierzanowski@wum.edu.pl

\begin{abstract}
Introduction: Constipation is one of the most frequent clinical and nursing problems both in palliative care patients and in nursing home residents.

Aim: To assess the occurrence of constipation and its risk factors in adult inpatient palliative care units versus nursing homes. Material and methods: An epidemiological study was performed in an inpatient hospice and a nursing home.

Results: Fifty-one hospice patients and 49 nursing home residents were included in the study. Cancer was the main clinical condition in $90 \%$ of the palliative care patients (PCPs), and dementia or other psychotic disorders were predominant in the nursing home residents (NHRs). More PCPs had constipation than did NHRs ( $80 \%$ vs. $59 \% ; p=0.02$ ), although none of the single constipation symptoms differed statistically between these two groups. The insufficient food intake was twice as severe in the hospice patients $(p=0.0001)$. $68.6 \%$ of PCPs took strong opioids, while none of the NHRs did. Three times more NHRs spent at least $50 \%$ of daytime in bed than did PCPs $(73.5 \%$ vs. $23.5 \%$; $p<0.0001)$.

Conclusions: Constipation is very frequent in both palliative care patients and nursing home residents, but PCPs are more prone to it. The NHR and PCR groups should not be treated uniformly as the end-of-life population, referring to prevention and treatment of constipation, therapy needs, and the means enrolled for optimal symptom control.
\end{abstract}

\section{Introduction}

Constipation is one of the most frequent clinical and nursing problems. In the general population of Europe, the mean value of the reported constipation rates is $17.1 \%$, with a wide range from $0.7 \%$ to $81 \%$ due to different definitions of constipation assumed in the studies. Aging is the most crucial risk factor for constipation; while in children this symptom is rare, one-third of the people over 70 years old complains of it. In people older than 75 years, constipation is twice as frequent as in those under 75 years of age. Similarly, in demented patients, it is two-fold more frequent [1]. It is two-fold more frequent in women and almost twice as often in the low socioeconomic status population compared to highly educated persons (10.2\% vs. $6.3 \%$, respectively). Various other risk factors are associated with constipa- tion, such as dietary habits (e.g. consumption of olive oil and meat), lifestyle factors (living in a large community or retirement home and immobility), pregnancy, frequency of breastfeeding, waist/hip ratio, anxiety and depression, clinical condition (comorbidities, other bowel disorders, previous hysterectomy and posterior colpocele), and the use of various medications including laxatives [2-4].

Bowel function may worsen as people become more unwell. The group particularly exposed to persistent constipation are patients with cancer or other advanced, progressive illness, especially those taking opioid analgesics. That is why constipation is reported in $42.4 \%$ of palliative care patients, and it is one of the most frequent somatic symptoms, besides fatigue, pain, and cachexia. The proportion of people with se- 
vere problems increases as death approaches, despite the time and number of interventions currently used to palliate these problems [5, 6].

Except for advanced cancer, the population treated in palliative inpatient care units is quite similar regarding age, concomitant morbidities, and nursing needs to that in nursing homes. These two populations are often collected in one notion of the end-of-life persons, which in our opinion might be misleading.

Constipation is a frequent and bothersome symptom in nursing home residents, as well as in hospice patients [7]. The additional burden of progressive life-threatening disease, as well as the treatment and side effects of the medications used in palliative care patients, entails an increase in the prevalence of common symptoms like constipation. The comparison of these two cohorts could give some clarification of the specificity and incremental treatment and nursing needs of palliative care patients.

\section{Aim}

This study was performed to assess the difference in the occurrence of constipation and its risk factors in inpatient palliative care units and nursing homes.

\section{Material and methods \\ Inclusion criteria}

The inclusion criteria were:

1. Adults (18 years and more).

2. Karnofsky performance status of 20 and more.

3. Aware and able to complete a routine examination. No exclusion criteria were set because the assessment was a part of a routine examination.

\section{Data collection procedure}

The subjective examination of the palliative care unit patients and the nursing home residents was performed by one trained nurse using a questionnaire to ensure standardised and comparable results. The assessment was a part of a nurse routine mandatory assessment and reassessment. The assessing nurse did not analyse the data and did not know the statistical results before the end of the data collection time. All data collected (assessments, ratings, and measurements) refer to the mean values for the last seven days until the assessment day.

The sample size was estimated at 40 persons in each group to achieve $80 \%$ power of the study.

The mandatory information on constipation symptoms collected by the nurse include: Karnofsky Performance Status (rated by the nurse), days since the last bowel movement, the frequency of bowel movements (the total number of days with at least one effective defecation during the last 7 days), difficulty with defecation, stools too hard, straining during defecation, necessity of the laxatives, patient's subjective assessment of constipation.

Subjective symptoms were assessed by the patients using the 5 -point rating scale (0-4), where 0 means no symptom and 4 means maximal intensity/severity.

The risk factors assessed were: bed rest (immobilisation) - the average ratio (\%) of daytime spent in bed, insufficient fluid intake, insufficient food intake, inadequate privacy during defecation, dependence on caregivers.

Insufficient fluid intake and insufficient nutrition refer to the amounts of fluids and food taken by the patients on his own or with help, and were assessed using the 5-point rating scale:

0 - no insufficiency,

1 - insignificant nutrition/fluid intake impairment (some attention was required to provide adequate nutrition/fluid intake),

2 - moderate nutrition/fluid intake impairment,

3 - major nutrition/fluid intake impairment (fluids/food taken with effort),

4 - significant insufficiency; based on caregiving nurses' assessment (not a calculation of real water or caloric intake).

The privacy conditions during bowel movements were rated by the assessing nurse, based on the observation of the environment conditions; the 5-point rating scale was used:

0 - privacy ensured,

1 - privacy ensured, but others' assistance was required after defecation,

2 - privacy was impaired by the presence of other patients or persons in the room during defecation,

3 - privacy was impaired by the need for assistance during defecation,

4 - no privacy ensured (e.g. an open door during defecation). The patient's perception may differ from the assessment of the nurse.

The dependence on caregivers was assessed using the five-point rating scale, where:

0 - full independence (self-service),

1 - assistance for some activities necessary (e.g. moving to the toilet),

2 - hygienic activities need assistance,

3 - most of the simple daily activities need assistance,

4 - full dependence (no self-service possible).

\section{Ethics}

The Ethics Committee of the Medical University of Lodz approved the study. 
Table I. Demographic characteristics of the studied population

\begin{tabular}{|c|c|c|c|}
\hline Parameter & $\begin{array}{l}\text { Nursing home }(95 \% \mathrm{Cl}) \\
(n=49)\end{array}$ & $\begin{array}{l}\text { Inpatient hospice }(95 \% \mathrm{Cl}) \\
(n=51)\end{array}$ & $P$-value \\
\hline Age, mean (range) & $71.3(58.8-90.0)$ & $66.3(54.9-83.3)$ & 0.087 \\
\hline Female gender (\%) & 59.2 & 45.1 & 0.168 \\
\hline $\begin{array}{l}\text { Overall performance status } \\
\text { (Karnofsky 0-100), mean (range) }\end{array}$ & $53.3(45.0-65.6)$ & $42.7(32.3-58.3)$ & 0.00001 \\
\hline
\end{tabular}

\section{Statistical analysis}

Frequency analysis was performed using the $\chi^{2}$ test and Fisher's exact test. The Mann-Whitney $U$ test for the nonparametric data to compare two sample means.

$P$-values less than 0.05 were considered statistically significant, and the Bonferroni correction was applied for multiple comparisons.

The Data was analysed by Statistica 13 (StatSoft).

\section{Results}

\section{Demographic characteristics}

Data of a total of 100 patients were enrolled in the analysis: 51 inpatient palliative care patients (PCP group) and 49 nursing home residents (NHR group). The groups did not differ regarding age and sex (Table I) The PCPs' overall performance status was significantly lower (45\%) than that of the NHRs (59\%).

Cancer was the main clinical condition in $90 \%$ of the palliative care patients. In the nursing home residents, the main diagnosis was dementia and other psychotic disorders, and only 4\% (2 persons) had a neoplasm (Table II).

\section{Occurrence and symptoms of constipation}

Constipation was diagnosed in $59.2 \%$ of the nursing home residents and $80.4 \%$ of the palliative care patients $(p=0.02)$. However, none of the elements of the assumed definition of constipation differed between the groups NHR and PCP (Table III). The mean frequen-
Table II. The main diagnosis of the studied cohort

\begin{tabular}{lcc} 
Diagnosis & $\begin{array}{c}\text { Nursing home } \\
(n=49)(\%)\end{array}$ & $\begin{array}{c}\text { Inpatient } \\
\text { hospice } \\
(n=51)(\%)\end{array}$ \\
\hline Other & 40.8 & 9.8 \\
\hline Cancer & 4.1 & 90.2 \\
\hline Neurological disease & 16.3 & 0.0 \\
\hline Psychotic disorders & 38.8 & 0.0
\end{tabular}

cy of bowel movements was 4.2 and 3.1 in the NHR and PCP groups, correspondingly $(p=0.03)$, but taking the Bonferroni correction into account, the difference should be analysed with caution.

\section{Risk factors}

Bed rest (immobilisation) assessed as the percentage of daytime was significantly more prevalent in the nursing home participants than in those from the inpatient hospice. Mean bed rest values were $49 \%$ and $22.5 \%$ in the NHR and PCP groups, respectively ( $p=$ 0.00002 ). The ratio of persons spending at least $50 \%$ of daytime in bed was $73.5 \%$ and $23.5 \%$ in the NHR and PCP groups, respectively $(p<0.0001)$.

The mean intensity of risk factors for constipation related to nursing is presented in Figure 1 . The mean insufficient food intake factor is twice as severe in the

Table III. Symptoms of constipation

\begin{tabular}{|c|c|c|c|c|c|}
\hline \multirow[t]{2}{*}{ Symptoms } & \multicolumn{2}{|c|}{ Nursing home } & \multicolumn{2}{|c|}{ Inpatient hospice } & \multirow[t]{2}{*}{$P$-value } \\
\hline & Mean & $N$ & Mean & $N$ & \\
\hline Bowel movements (in the last 7 days) & 4.2 & 49 & 3.1 & 51 & 0.030 \\
\hline Difficulty of defecation & 1.6 & 49 & 1.9 & 51 & 0.213 \\
\hline Feeling of incomplete defecation & 1.3 & 49 & 1.3 & 51 & 0.975 \\
\hline Stools too hard & 1.6 & 49 & 1.6 & 51 & 0.889 \\
\hline Straining & 1.5 & 49 & 1.9 & 51 & 0.188 \\
\hline Necessity of laxatives & 1.3 & 49 & 1.7 & 51 & 0.176 \\
\hline Assessment of constipation & 1.7 & 49 & 1.8 & 51 & 0.689 \\
\hline
\end{tabular}




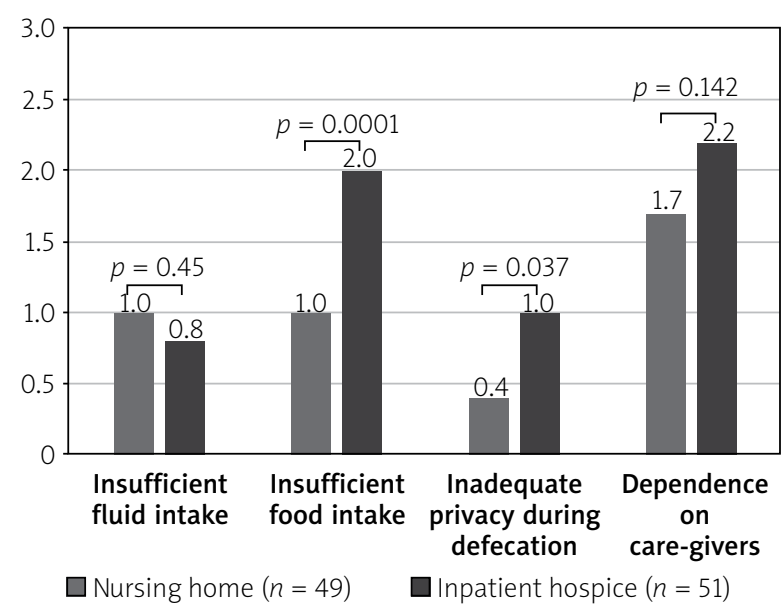

Figure 1. Nursing risk factors

hospice patients $(p=0.0001)$. There is no statistical difference between groups for insufficient fluid intake and dependence on caregivers. Inadequate privacy during defecation is more expressed in the inpatient hospice, but after Bonferroni correction for multiple comparisons the difference is not statistically significant or should be analysed with caution $(p=0.037)$.

\section{Concomitant symptoms}

The most frequent symptoms were weakness (100\%), cachexia and sleeping disorders ( $82 \%$ each), chronic pain (70\%), depression (65\%), and dyspnoea $(57 \%)$ in the hospice patients, while in the nursing home residents these were: weakness (65\%), sleeping disorders (49\%), and depression (38\%).

The Figure 2 depicts the pattern of the mean intensity of the most common symptoms reported in the hospice patients and its comparison to the nursing home residents. Except for diarrhoea, all symptoms were more frequent in hospice patients than in nursing homes residents. However, the differences for anxiety and pruritus are statistically insignificant. Taking Bonferroni correction into account, the differences for sleeping disorders, depression, and dyspnoea should also be considered with caution.

\section{Opioid analgesic consumption}

Two out of the 49 nursing home residents were taking weak opioids (tramadol), while 35 (68.6\%) of the hospice patients were treated with at least one strong opioid.

\section{Discussion}

The population of the elderly is growing. Along with aging, the percentage of people suffering from chronic diseases increases, as well as the incidence of neoplasms. Palliative care patients differ from nursing homes residents by being afflicted by cancer or other end-stage clinical conditions. Thus, comparing the symptomatology in these two populations may reflect the real impact on the clinical status of the additional burden of cancer and its treatment.

Gastrointestinal symptoms are among the most frequent and troublesome in palliative care patients. Constipation is particularly difficult to prevent and often refractory to traditional laxatives. There are usually many concomitant causes of constipation in these patients, among which the behavioural factors, such as immobilisation, disability, and insufficient fluid and food intake, or dependence on the caregivers, seem to be essential in the aetiology of occurrence and degree of constipation [8, 9]. Although several drugs may cause constipation, opioid analgesics, used commonly in the treatment of cancer pain, are regarded as the most important risk factor [10-12].

The palliative care and nursing home cohorts did not differ from each other in this study regarding gen-

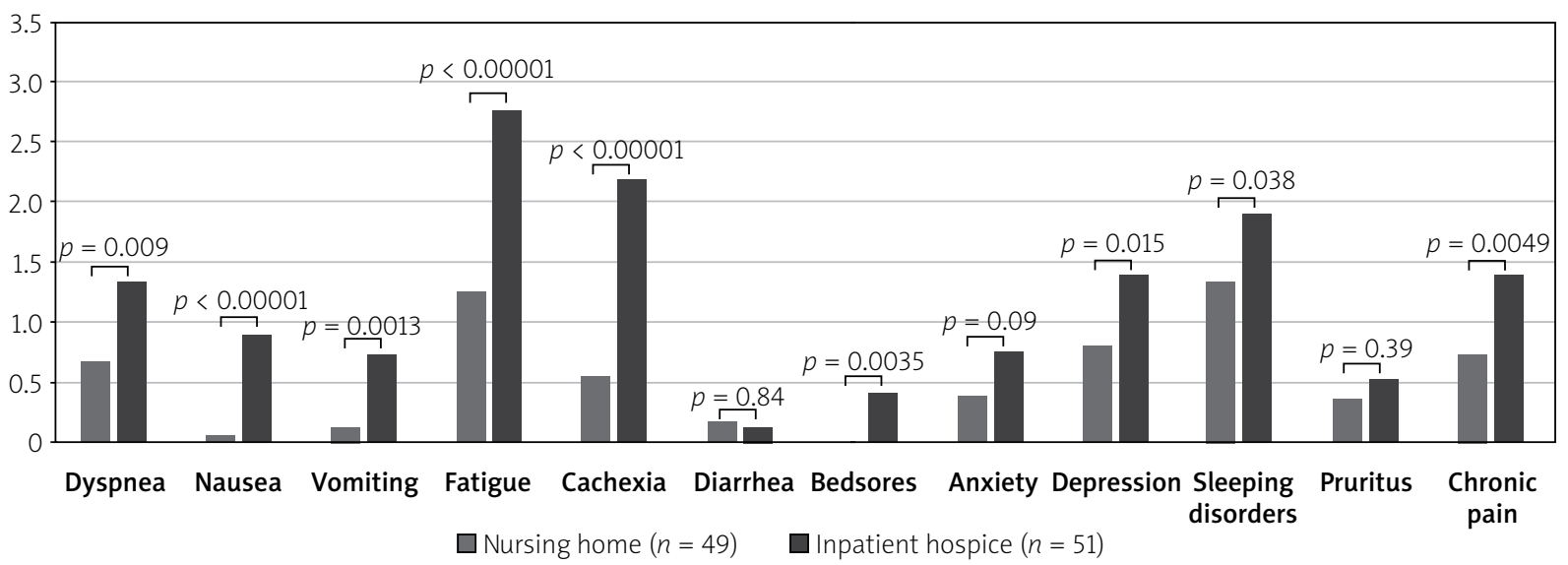

Figure 2. The mean intensity of the most common symptoms in nursing home residents and inpatient hospice patients 
der and age. The Karnofsky overall performance status was significantly lower in the palliative patients, as they were affected by cancer. In our previous study, this was an independent risk factor for constipation [13]. The vast majority (90\%) of the hospice patients had a neoplasm, while this was rare among the nursing home residents. The predominant diseases in the latter population were psychotic and neurological disorders (dementia, post-stroke conditions).

There is no commonly agreed definition of constipation. It typically consists of both objective and subjective symptoms. There is no universally accepted definition of constipation, and the Rome Criteria (III and IV) are not useful in the short life-expectancy population, which we addressed in previous studies [14-16]. In this study, we assumed the definition that follows the recommendations of the Polish Society of Palliative Medicine, i.e. decreased frequency of bowel movements (less than three per week), or subjective symptoms such as the difficulty with defecation, overly hard stools, straining during defecation, the necessity of the use of laxatives, or subjective patient's assessment of constipation. For the diagnosis of constipation, one of the above symptoms was necessary (less than three bowel movements per week or intensity of at least one subjective symptom over 1 in a 0-4 scale).

Using this definition, the majority of the hospice patients (80\%), as well as nursing home residents (59\%), had constipation, and the difference between groups is statistically significant. These figures are much higher than in the systematic review that we referred to (42\%) [5]. In our opinion, there are several reasons for that. One is the more rigorous definition taken by us. We must assume the possibility of bias in data collection done by one person only. However, we pointed out that the traditional understanding of constipation is unsuitable for the palliative care population because these patients are frailer and even a small decrease in frequency of bowel movements can cause substantial worsening of subjective symptoms [15]. The last, but still very probable, reason is that the patients in this study were in a more advanced stage of disease, and so were the residents of the nursing home.

It is interesting that in spite of the difference in the occurrence of constipation between the studied groups, the frequency of no single symptom differed statistically, maybe except for the frequency of bowel movements; this suggests that the probably a diagnosis of constipation should be based on several precisely defined statements rather than on a common understanding of the notion.

Immobilisation and low physical activity are commonly meant to be factors for constipation. However, we did not prove that bed rest is a risk factor for constipation in our previous study with 237 patients ( $p=$ 0.046 assumed for statistical significance) [13]. Over three times more nursing home residents are immobilised for at least $50 \%$ of the day than were the hospice patients. The mean bed rest ratio was two-fold higher in the nursing home residents. It seems that the importance of this factor may not be very high in the multifactorial aetiology. That is why, although the nursing home residents were much more often bed-resting, the occurrence of constipation did not correlate with this. It is worth mentioning on the fact that although the NHRs spent more time in bed than the PCPs, they were in relatively better general condition. This paradoxical inconsistency may be explained by taking into consideration the fact that the NHRs are mostly demented persons with little will to undertake any physical activity, while many PCPs are more likely to partake in activities of daily living, independently or with the help of a caregiver, despite their disability.

The mean food intake was twice as bad in the palliative care patients. It is comprehensive because cachexia and anorexia commonly accompany malignancy. They are unquestioned risk factors for constipation, but they are very difficult to manage and even impossible to reverse in refractory phase [17]. The dietary interventions are hardly possible in many cases because, along with worsening of the clinical condition of patients, the appetite and the physical abilities to eat are also gradually deteriorating. Sufficient food supply is in many cases difficult or even futile.

The second important nursing risk factor for constipation is insufficient fluid intake. In this study, we did not observe any statistical difference between hospice patients and nursing home residents. Both oral and parenteral water supplementation are provided in these institutions with care. That is also true for the relatively good assistance of the caregivers and privacy conditions during defecation. However, these results are specific to the institutions involved, not as a general conclusion.

We have assessed the occurrence of concomitant diseases, and we found the patterns typical for both populations. Although the most prevalent symptoms are similar and weakness, sleeping disorders, and depression are frequent common, the populations differ very much. Not only are some symptoms specific to the palliative care patients, but also the intensity of all symptoms is much higher than in the long-term care residents. It is important to understand that these two populations differ in clinical conditions and needs, survival trajectories, and the means enrolled for optimal symptom control. 
One of the most important constipating factors is opioid analgesics, which produce opioid bowel dysfunction syndrome (OBD). It is the most frequent adverse effect of opioid treatment, which affects $70-90 \%$ of these patients, as well as other concomitant causes of constipation in palliative care patients [18, 19]. In contrast to other transient symptoms like somnolence, nausea, and vomiting, constipation does not cease but increases with the length of opioid treatment [20]. None of the nursing home residents received strong opioids, while two-thirds of the hospice patients did. It is probable that in the latter cohort constipation develops more easily.

\section{Conclusions}

Palliative care patients and nursing home residents are populations similar in age, gender, and many symptoms related to the frailty, but they differ in their therapy needs. Constipation and other concomitant symptoms are much more common in the hospice patients. Cancer and its treatment bring additional symptom burden that seems difficult to manage. The two populations cannot be treated in the same manner, and much more effort and resources are expected to be necessary for palliative care patients.

\section{Acknowledgments}

We thank Mrs. Anna Dzwonnik for her invaluable help in data collection.

\section{Conflict of interest}

The authors declare no conflict of interest.

\section{References}

1. Talley NJ, Jones M, Nuyts G, Dubois D. Risk Factors for chronic constipation based on a general practice sample. Am J Gastroenterol 2003; 98: 1107-11.

2. Bytzer P, Howell S, Leemon M, et al. Low socioeconomic class is a risk factor for upper and lower gastrointestinal symptoms: a population based study in 15000 Australian adults. Gut 2001; 49: 66-72.

3. Peppas G, Alexiou VG, Mourtzoukou E, Falagas ME. Epidemiology of constipation in Europe and Oceania: a systematic review. BMC Gastroenterol 2008; 8: 5.

4. Dzierżanowski T, Rydzewska G. Constipation - a difficult therapeutic problem. Gastroenterology Rev 2012; 7: 249-63.

5. Clark K, Smith JM, Currow DC. The prevalence of bowel problems reported in a palliative care population. J Pain Symptom Manage 2012; 43: 993-1000.

6. Potter J, Hami F Bryan T, Quigley C. Symptoms in 400 patients referred to palliative care services: prevalence and patterns. Palliat Med 2003; 17: 310-4.

7. Blekken LE, Nakrem S, Vinsnes AG, et al. Constipation and laxative use among nursing home patients: prevalence and as- sociations derived from the residents assessment instrument for Long-Term Care Facilities (interRAI LTCF). Gastroenterol Res Pract 2016; 2016: 1215746.

8. Chin A Paw MJ, van Poppel MN, van Mechelen W. Effects of resistance and functional-skills training on habitual activity and constipation among older adults living in long-term care facilities: a randomized controlled trial. BMC Geriatrics 2006; 6: 9.

9. Iovino P, Chiarioni G, Bilancio G, et al. New onset of constipation during long-term physical inactivity: a proof-of-concept study on the immobility-induced bowel changes. PLoS One 2013; 8: e72608.

10. Sykes NP. The relationship between opioid use and laxative use in terminally ill cancer patients. Palliat Med 1998; 12: 375-82.

11. Dzierżanowski T. Opioid induced bowel dysfunction in cancer patients. Med Paliat 2012; 2: 57-66.

12. Richmond JP, Wright ME. Development of a constipation risk assessment scale. J Orthop Nurs 2006; 10: 186-97.

13. Dzierżanowski T, Ciałkowska-Rysz A. Behavioral risk factors of constipation in palliative care patients. Support Care Cancer 2015; 23: 1787-93.

14. Lembo A, Camilleri M. Chronic constipation. N Engl J Med 2003; 349: 1360-8.

15. Dzierżanowski T, Ciałkowska-Rysz A. Assessment of the usefulness of the definition of constipation in palliative care patients according to Polish Society of Palliative Medicine. Med Paliat 2011; 1: 33-8.

16. Longstreth GF, Thompson WG, Chey WD, et al. Functional bowel disorders. Gastroenterology 2006; 130: 1480-91.

17. Fearon K, Strasser F, Anker SD, et al. Definition and classification of cancer cachexia: an international consensus. Lancet Oncol 2011; 12: 489-95.

18. Lucero M, Von Scheele B, Blackard R, et al. The incidence and impact of gastrointestinal adverse events (GIAEs) associated with opioid analgesic use: a review of the literature. J Pain 2006; 7: S89.

19. Mercadante S, Villari P, Ferrera P, Casuccio P. Opioid-induced or pain relief-reduced symptoms in advanced cancer patients? Eur J Pain 2006; 10: 153-9.

20. Annunziata K, Freedman D, Janning S, et al. Constipation is a predominant side effect of opioid treatment for persistent pain. J Pain 2006; 7 Suppl: S89.

Received: 21.02.2018

Accepted: 25.04 .2018 